\title{
Context-Aware Neural Model for Temporal Information Extraction
}

\author{
Yuanliang Meng \\ Text Machine Lab for NLP \\ Department of Computer Science \\ University of Massachusetts Lowell \\ ymeng@cs.uml . edu
}

\author{
Anna Rumshisky \\ Text Machine Lab for NLP \\ Department of Computer Science \\ University of Massachusetts Lowell \\ arumecs.uml .edu
}

\begin{abstract}
We propose a context-aware neural network model for temporal information extraction, with a uniform architecture for event-event, event-timex and timex-timex pairs. A Global Context Layer (GCL), inspired by the Neural Turing Machine (NTM), stores processed temporal relations in the narrative order, and retrieves them for use when the relevant entities are encountered. Relations are then classified in this larger context. The GCL model uses long-term memory and attention mechanisms to resolve long-distance dependencies that regular RNNs cannot recognize. GCL does not use postprocessing to resolve timegraph conflicts, outperforming previous approaches that do so. To our knowledge, GCL is also the first model to use an NTM-like architecture to incorporate the information about global context into discourse-scale processing of natural text.
\end{abstract}

\section{Introduction}

Extracting information about the order and timing of events from text is crucial to any system that attempts an in-depth natural language understanding, whether related to question answering, temporal inference, or other related tasks. Earlier temporal information extraction (TemporalIE) systems tended to rely on traditional statistical learning with feature-engineered task-specific models, typically used in succession (Yoshikawa et al., 2009; Ling and Weld, 2010; Sun et al., 2013; Chambers et al., 2014; Mirza and Minard, 2015).

Recently, there have been some attempts to extract temporal relations with neural network models, particularly with recurrent neural networks
(RNN) models (Meng et al., 2017; Cheng and Miyao, 2017; Tourille et al., 2017) and convolutional neural networks (CNN) (Lin et al., 2017). These models predominantly use token embeddings as input, avoiding handcrafted features for each task. Typically, neural network models outperform traditional statistical models. Some studies also try to combine neural network models with rule-based information retrieval methods (Fries, 2016). These systems require different models for different pair types, so several models must be combined to fully process text.

A common disadvantage of all these models is that they build relations from isolated pairs of entities (events or temporal expressions). This context-blind, pairwise classification often generates conflicts in the resulting timegraph. Common ways of ameliorating the conflicts is to apply some ad hoc constraints to account for basic properties of relations (e.g. transitivity), often without considering the content of the text per se. For example, Ling and Weld (2010) designed transitivity formulae, used with local features. Sun (2014) proposed a strategy that "prefers the edges that can be inferred by other edges in the graph and remove the ones that are least so". Another approach is to use the results from separate classifiers to rank results according to their general confidence (Mani et al., 2007; Chambers et al., 2014). High-ranking results overwrite low-ranking ones. Meng et al. (2017) used a greedy pruning algorithm to remove weak edges from the timegraph until it is coherent.

When humans read text, we certainly do not follow the procedure of interpreting interpret relations only locally first, and later come up with a compromise solution that involves all the entities. Instead, if local information is insufficient, we consider the relevant information from the wider context, and resolve the ambiguity as soon as possible. The resolved relations are stored in our 
memory as "context" for further processing. If the later evidence suggests our early interpretation was wrong, we can correct it.

This paper proposes a model to simulate such mechanisms. Our model introduces a Global Context Layer (GCL), inspired by the Neural Turing Machine (NTM) architecture (Graves et al., 2014), to store processed relations in narrative order, and retrieve them for use when related entities are encountered. The stored information can also be updated if necessary, allowing for self-correction.

This paper's contributions are as follows. To our knowledge, this is the first attempt to use neural network models with updateable external memory to incorporate global context information for discourse-level processing of natural text in general and for temporal relation extraction in particular. It gives a uniform treatment of all pairs of temporally relevant entities. We obtain stateof-the-art results on TimeBank-Dense, which is a standard benchmark for TemporalIE.

\section{Dataset}

We train and evaluate our model on TimeBankDense $^{1}$ (Chambers et al., 2014). There are 6 classes of relations: SIMULTANEOUS, BEFORE, AFTER, IS_INCLUDED, INCLUDES, and VAGUE TimeBank-Dense annotation aims to approximate a complete temporal relation graph by including all intra-sentential relations, all relations between adjacent sentences, and all relations with document creation time. TimeBank-Dense is one of the standard benchmarks for intrinsic evalution of TemporalIE systems. We follow the experimental setup in Chambers et al. (2014), which splits the corpus into training/validation/test sets of 22,5 , and 9 documents, respectively. Previous publications often use the micro-averaged F1 score, which is equivalent to accuracy in this case. We also rely on the micro-averaged F1 score for model selection and evaluation.

Following Meng et al. (2017), we augment the data by flipping all pairs, except for relations involving document creation time (DCT). In other words, if a pair $\left(e_{i}, e_{j}\right)$ exists, we add $\left(e_{j}, e_{i}\right)$ to the dataset with the opposite label (e.g. BEFORE becomes AFTER). The augmentation applies to the validation and test sets also. In the final evaluation, a double-checking technique picks one result from

\footnotetext{
${ }^{1}$ https: / / www. usna. edu/Users/cs / nchamber/caevo/\#corpus
}

the two-way classification, based on output scores. The dataset is heavily imbalanced. The training set has as much as $44.1 \%$ VAGUE labels, whereas only $1.8 \%$ labels are SIMULTANEOUS. We did not do any up-sampling or down-sampling.

\section{System}

Our system has two main components. The first one is a pairwise relation classifier, and the other is the Global Context Layer (GCL). The pairwise relation classifier follows the architecture designed by Meng et al. (2017), which used the dependency paths to the least common ancestor (LCA) from each entity as input. We train the first component first, and then assemble them in a combined neural network to continue training. Fig. 1 gives an overview of the system.

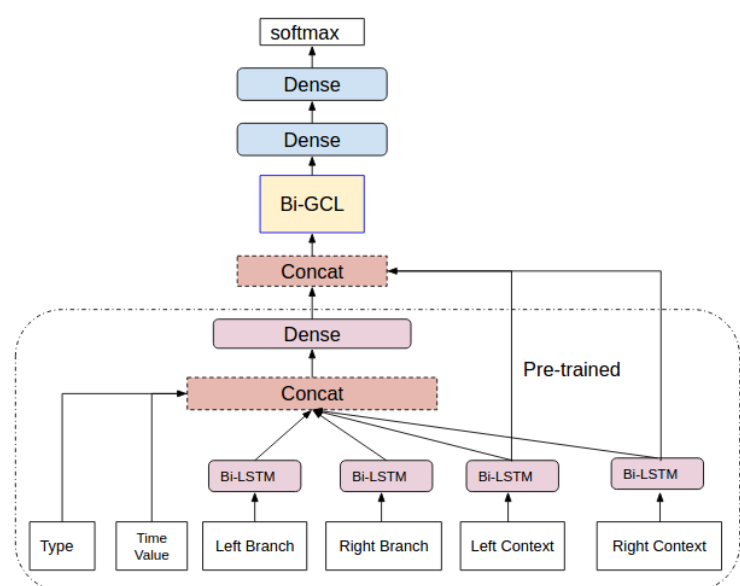

Figure 1: System overview. Originally, the pre-trained system has one more dense layer and an output layer, but they are truncated before combination. The max pooling layers on top of each Bi-LSTM layers are omitted here.

\subsection{Global Context Layer}

The Global Context Layer (GCL) we propose is inspired by the Neural Turing Machine (NTM) architecture, which is an extension of a recurrent neural network with external memory and an attention mechanism for reading and writing to that memory. NTM has been shown to perform basic tasks such as copying, sorting, and associative recall (Graves et al., 2014). The external memory not only enables a large (theoretically infinite) capacity for information storage, but also allows flexible access based on attention mechanisms.

Essentially, GCL is a specialized form of NTM, which eliminates some parameters to facilitate training, and specializes some functions to impose restrictions. While not as powerful as the canoni- 
cal NTM, it is more suitable for the task of retaining and updating global context information.

\subsubsection{Motivation}

Vanilla RNNs struggle with capturing longdistance dependencies. Gated RNNs such as LSTM have trainable gates to address the "vanishing and exploding gradient" problem (Hochreiter and Schmidhuber, 1997). At each time step, it chooses what to memorize and forget, so patterns over arbitrary time intervals can be recognized. However, the memory in LSTM is still short-term. No matter how long the cell states keep certain information, once it is forgotten, it gets lost forever. Such a mechanism suffices for modeling contiguous sequences. For example, sentences are naturally fit units for such models, since a sentence starts only after the preceding sentence is finished, and LSTM may be an adequate tool to process sentences. However, when the sequences are not contiguous, as in temporal and other discourse-scale relations, LSTM models do not have the capability to look for input pieces across sequences.

When humans read text, discourse-level information is often distributed across the full scope of the text. To fully understand an article, we must be able to organize the processed information across sentences and paragraphs. In particular, to interpret temporal relations between entities in a sentence, sometimes we also look at relations with other entities elsewhere in the text. Such entities or relations form no regular sequences, and only a system with long-term memory as well as attention mechanisms can process them. An NTM-like architecture has an external memory with attention mechanisms, so it is an ideal candidate for such tasks. Furthermore, unlike the models that use attention over inputs (Vinyals et al., 2015; Kumar et al., 2016), NTM-like models are capable of updating previously stored representations. We describe below the GCL architecture that we use to store and update the global context information.

\subsubsection{Reading}

The input to the GCL layer is a concatenation of three layers from the pairwise neural network. Two of these are the entity context representation layers, encoded by the two LSTM branches. The other is the penultimate hidden layer before the output layer, which encodes the relation. We can write them as $\left[\mathbf{e}_{\mathbf{1}}, \mathbf{e}_{2}, \mathbf{x}\right]$. The context representations are used as "keys" to uniquely identify the

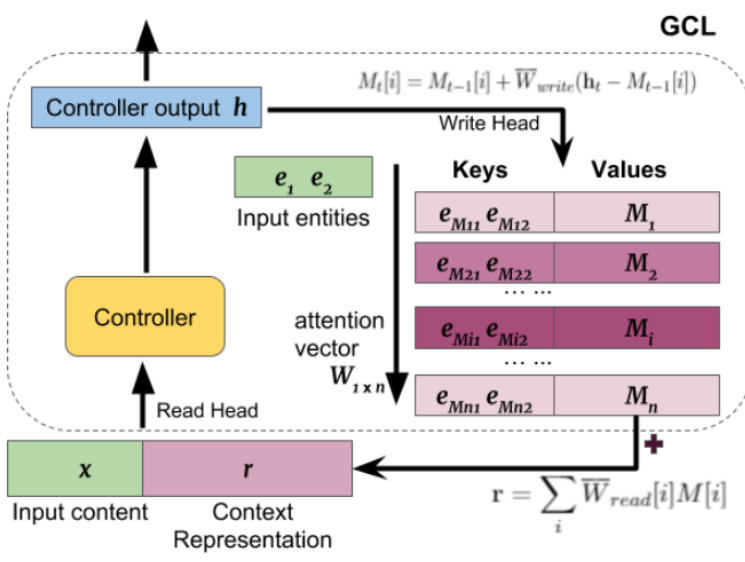

Figure 2: GCL computing attention weights. Input entity representations are compared to the Key section of GCL memory. Slots with the same or similar entities get more attention.

entities. Note that we use flat context embeddings, rather than dependency path embeddings, because dependency paths tend to be short and will also vary for the same entity, depending on the other entity in the pair. As such, they do not provide a unique way to represent an entity.

The original design of NTM has a complex addressing mechanism for reading, which also makes it difficult to train. An important difference in GCL is that we separate the "key" component from the "content" component of memory. Each memory slot $S[i]$ consists of $[K[i] ; M[i]]$, where $S$ is the whole memory with $n$ slots, $i \leq n$ is the index, $K$ is the key and $M$ is the content. Addressing is only performed on the key component. The key component stores the representation of two entities, provided by the layers encoding the flat entity context.

$$
K[i]=\mathbf{e}_{\mathbf{M 1}}[i] \oplus \mathbf{e}_{\mathbf{M} 2}[i]
$$

Here $\oplus$ is the concatenation operator. In the GCL model, the read head computes a reading weight $W_{n \times 1}$ from the input entity representations $\mathbf{e}_{1}, \mathbf{e}_{2}$ and the entity representations $\mathbf{e}_{\mathbf{M} 1}, \mathbf{e}_{\mathbf{M} 2}$ in memory (i.e., the keys in each memory slot). The first step is to compute the distance between current input and the memory columns, as shown in Eq. 2. $D[i]$ is the Euclidean distance between the input key and the memory key of slot $M[i] . \quad D^{\prime}[i]$ is computed after flipping the two entities. We do so because the order of entities in a pair should not affect their relevance.

$$
\begin{aligned}
D[i] & =\frac{1}{Z}\left\|\mathbf{e}_{\mathbf{1}} \oplus \mathbf{e}_{\mathbf{2}}-\mathbf{e}_{\mathbf{M} \mathbf{1}}[i] \oplus \mathbf{e}_{\mathbf{M} \mathbf{2}}[i]\right\|_{2}^{2} \\
D^{\prime}[i] & =\frac{1}{Z^{\prime}}\left\|\mathbf{e}_{\mathbf{2}} \oplus \mathbf{e}_{\mathbf{1}}-\mathbf{e}_{\mathbf{M} \mathbf{1}}[i] \oplus \mathbf{e}_{\mathbf{M} \mathbf{2}}[i]\right\|_{2}^{2}
\end{aligned}
$$


where $Z=\sum_{i} D[i]$ is the normalization factor, and so is $Z^{\prime}$ for the flipped case. The reading weight is then calculated as in Eq. 3 , where $\mathbf{1}_{n \times 1}$ is a vector of all 1's.

$$
\begin{array}{r}
W[i]=\max (\operatorname{softmax}(\mathbf{1}-\mathbf{D})[i], \\
\left.\operatorname{softmax}\left(\mathbf{1}-\mathbf{D}^{\prime}\right)[i]\right)
\end{array}
$$

Every element of $W$ represents the relevance of the corresponding memory slot (see Fig. 2). Often it is still too blurred and needs to be further sharpened as in Eq. 4. Here $\beta$ is a positive number. $W^{\beta}$ is a point-wise exponential function by power of $\beta$. A large $\beta$ allows "winner takes all", so only the most relevant memory slots are read.

$$
\bar{W}_{\text {read }}=\operatorname{softmax}\left(W^{\beta}\right)
$$

Parameter $\beta$ could be a constant, or could be trainable. Our model computes it from the current input $\mathbf{x}_{t}$ and the previous output $h_{t-1}$, and thus it varies in each time step. $W_{\text {sharp }}$ and $b_{\text {sharp }}$ are trainable weights and bias, $c_{\beta}$ is a constant, and $R e L U$ is the rectified linear function.

$$
\beta_{t}=\operatorname{Re} L U\left(W_{\text {sharp }}\left[\mathbf{x}_{t}, \mathbf{h}_{t-1}\right]+b_{\text {sharp }}\right)+c_{\beta}
$$

With the sharpened reading weight vector, we are able to obtain the read vector $\mathbf{r}_{1 \times m}$ from $M$ as a weighted sum, as in Eq. 6.

$$
\mathbf{r}=\sum_{i} \bar{W}_{\text {read }}[i] M[i]
$$

Generally speaking, the depth of memory $M$ should be large enough to allow sparse encoding, so that crucial information is not lost after the summation. The read vector then contains contextual information relevant to current input. Both the read vector and the current input are fed to the controller, yielding GCL output. Unlike the canonical NTM, the CGL model does not have a trainable gate interpolating the $\bar{W}_{t}$ computed at time $t$, with $\bar{W}_{t-1}$ computed at previous time $t-1$. The weight vector is not passed to next time step, so the attention has no "inertia".

We tried two variants of the controller: (a) statetracking, with an LSTM layer, and (b) stateless, with a dense layer. An LSTM controller has an internal state, and also has gates to select input and output. If the input data and/or the read vector from $M$ have regular patterns with respect to time steps, an LSTM controller would be a better choice. For the specific task of temporal relation extraction, we saw no difference in performance.

\subsubsection{Writing}

The controller produces an output $h_{t}$, which is sent to the next layer and also used to update $M$. Similar to reading, the first step of writing procedure is to compute an attention weight vector over the slots of $M$. As described above, the reading procedure computes a weighted sum over slots of $M$. The writing procedure writes a weighted $h_{t}$ to each slot. The attention mechanism here is de facto a soft addressing mechanism. The slots with a higher attention value will be the addresses which will get more of an update.

The same weight vector $W$ computed as shown in Eq. 3 is used for writing. However, an additional operation is introduced for writing. Recall that the weights are computed from entity representations. If the input entities are $\mathbf{e}_{\mathbf{1}}$ and $\mathbf{e}_{2}$, the weight vector should have high values in the slots corresponding to $\mathbf{e}_{\mathbf{1}}$ and/or $\mathbf{e}_{2}$. But we may not always want relevant memory slots to be overwritten. Instead, additional information can be written to a different slot. Additionally, when $M$ is relatively empty, as at the beginning, the addressing mechanism may treat all slots equally, and uniformly update all slots in the same way. In this case we want the weight vector to shift each time, so $M$ can diversify fast.

Therefore we use a shift function similar to the canonical NTM. The idea is to compute a shifted weight vector $\widetilde{W}$ by convolving $W$ with a shift kernel $\mathbf{s}$ which maps a shift distance to a probability value. For example, $\mathbf{s}(-1)=0.2, \mathbf{s}(0)=0.5$, $\mathbf{s}(1)=0.3$ means the probabilities of shifting left, no shifting, and shifting right are $0.2,0.5,0.3$, respectively. Generally speaking, we want $\mathbf{s}$ to give zeros for most shift distances, so the shifting operation is limited to a small range.

$$
\widetilde{W}[i]=\sum_{j=0}^{n-1} W[j] \mathbf{s}[i-j]
$$

At each time step, the shift kernel depends on current input and output. If the allowed shift range is $[-\mathrm{s} / 2,+\mathrm{s} / 2]$, we train a weight $W_{s}$ and bias $b_{s}$ to calculate the shift weights $C_{s \times 1}$,

$$
C_{t}=\operatorname{softmax}\left(W_{s}\left[\mathbf{x}_{t}, \mathbf{h}_{t}\right]+b_{s}\right)
$$

Then the weights are mapped to a circulant kernel to perform the convolution in Eq. 7, the final output is $\widetilde{W}$.

Finally, the sharpening still needs to be applied. For the writing procedure, both addressing and 
shifting are "soft" in nature, and thus could yield a blurred outcome. Again, we train the weights to obtain a sharpening parameter $\gamma$ each time, and perform softmax over $W$.

$$
\gamma_{t}=\operatorname{Re} L U\left(W_{\text {sharp }}\left[\mathbf{x}_{t}, \mathbf{h}_{t}\right]+b_{\text {sharp }}\right)+c_{\gamma}
$$

$$
\bar{W}_{\text {write }}=\operatorname{softmax}\left(\widetilde{W}^{\gamma}\right)
$$

$\widetilde{W}^{\gamma}$ is the point-wise exponential function, over the shifted weight vector. $c_{\gamma}$ is a positive constant.

The original NTM model has gates for interpolating $\widetilde{W}^{\gamma}$ at the current time with the one computed at the previous time step, but we omit this operation. We also omit the erase vector and the add vector, so $\bar{W}_{\text {write }}$ fully controls what to overwrite in $M$ and what to retain. As a result, the writing operation can be expressed as:

$$
M_{t}[i]=M_{t-1}[i]+\bar{W}_{\text {write }}[i]\left(\mathbf{h}_{t}-M_{t-1}[i]\right)
$$

The first term in Eq. 11 is the memory in the previous time step, and the second term is the update. We update the keys in the same way. As we can see, the keys come from entity representations, but are not exactly the same, due to $\bar{W}_{\text {write }}$.

$$
K_{t}[i]=K_{t-1}[i]+\bar{W}_{\text {write }}[i]\left(\mathbf{e}_{\mathbf{1}} \oplus \mathbf{e}_{\mathbf{2}}-K_{t-1}[i]\right)
$$

\subsubsection{GCL vs. Canonical NTM}

We highlight below some major differences between the canonical NTM and the GCL model. Typically, NTM computes the keys from input and output for accessing different memory addresses. In GCL, the keys are simply the entity representations $\left[\mathbf{e}_{1}, \mathbf{e}_{2}\right]$ from input, in either order. The key function effectively involves slicing and flipping the input. Further discussion of the differences between the GCL addressing mechanism and some of the other NTM variations is provided in Sec. 5.

Another major difference is that we do not use any gates to interpolate the attention vector at the current time step with the one from the previous time step. Instead, the previous attention vector is totally ignored. Since we do not compute the erase vector or the add vector, this allows the attention vector to fully control memory updates.

In addition, we unified the trainable weights for calculating $\beta$ and $\gamma$ at each time step. We found these parameters not to be crucial, and setting them to be constant does not affect the results. We also do not shift attention for reading. A possible advantage of shifting attention is that neighboring slots of the focus can also be accessed, providing a way to simulate associative recall. This is based on the fact that the writing procedure tends to write similar memories close to each other. However, in this study we want the reading procedure to be restricted. Associative recall can be realized from attention vector itself, without shifting.

\subsection{Pairwise Classification Model}

The pairwise model classifies individual entity pairs, where entities are events and time expressions (timexes). In other words, for each pair, we only use the local context, and the relation of one pair does not affect the classification results for other pairs. We follow the architecture proposed in Meng et al. (2017), but with the following changes: (1) all three types of pairs are handled by the same neural network, rather than by three separately trained models; (2) the neighboring words (a flat context) of entity mentions are used to generate input, in addition to words on syntactic dependency paths; (3) all timex-timex pairs are included as well, not only event-timex and event-event pairs; (4) every pair is assigned a 3-dimensional "time value", to approximate the rule-based approach when possible.

\subsubsection{Event Pairs and Event-Timex Pairs}

TimeBank-Dense dataset labels three types of pairs: intra-sentence, cross-sentence and document creation time (DCT). For intra-sentence pairs and cross-sentence pairs, we follow Meng et al. (2017). The shortest dependency path between the two entities is identified, and the word embeddings from the path to the least common ancestor for each entity are processed by two LSTM branches, with a separate max pooling layer for each branch. Path to the root is used for cross-sentence relations. For relations with the DCT, we use a single word now as a placeholder for the DCT branch. Unlike Meng et al. (2017), we allow the model to accept all three pair types, with a "pair type" feature as a component of input, defined as an integer with the value $1,-1$ or 0 , respectively.

In addition to the shortest dependency path, our model also uses a flat local context window, that is, the words around each entity mention, regardless of syntactic structures. For an entity starting with word $w_{i}$, the local context window is 5 words to the left and 10 words to its right i.e. $w_{i-5} w_{i-4} \ldots w_{i} w_{i+1} \ldots w_{i+10}$. The windows are cut 
short at the edge of a sentence, or when the second entity in encountered. By using this context window, the words between two entities are often used twice by the system, and thus given more consideration. To inform the system of other entity mentions, we also add special input tokens at the locations where events and timexes are tagged. The embeddings of the special tokens are uniformly initialized, and automatically tuned during the training process.

\subsubsection{Timex Pairs}

The method described in Meng et al. (2017) classifies timex pairs by handcrafted rules and then adds them to the final results prior to postprocessing. Since timexes have concrete time values, a rule-based method would seem appropriate. However, since our model uses global context to help classify relations and timex-timex pairs enrich the global context representation, we design a way for a common classifier model to handle such pairs.

When DCT is not involved, timex pairs are created the same way as cross-sentence pairs, that is, path to the root is used for each entity. DCT is represented by the placeholder word now. In addition to the word-based representations, another input vector is used to simulate the rule-based approach, to be explained next.

\subsubsection{Time Value Vectors}

Every timex tag has a time value, following the ISO-8601 standard. Every value can be mapped to a 2D vector of real values (start, end). For a pair we use the subtraction of the vectors to represent the difference. Suppose we have timexes in below:

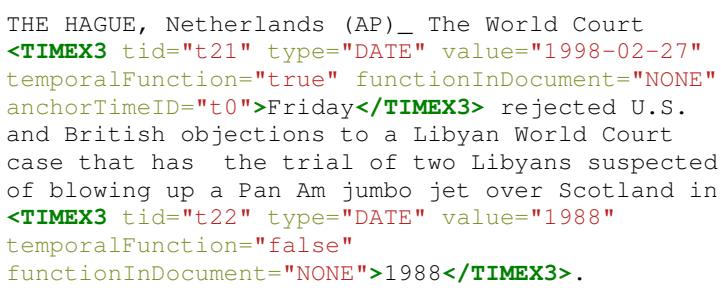

The first timex can be represented as $(1998+1 / 12$ $+26 / 365,1998+1 / 12+26 / 365)=(1998.155$, 1998.155), and the second one (1988, $1988+$ $364 / 365)=(1988,1988.997)$. The difference of the values are put in the sign function, to obtain the representation: $(\operatorname{sign}(1988-1998.155)$, $\operatorname{sign}(1988.997-1998.155))=(-1,-1)$. Vector $(-1$, -1) clearly indicates the AFTER relation between $t 21$ and $t 22$. We set the minimum interval to be a day, which is generally sufficient for our data. The
DURATION timexes are not considered, and wordbased input vectors are used to represent them.

In order to make all the input data have the same shape, we assign the time value vector to all pairs, even if a timex is not involved. For non-timex pairs, a vector $(-1,0,0)$ is used. The first element -1 to indicate a "pseudo" time value. Real timex pairs have the first value of 1 , so the example we just discussed would be assigned a vector $(1,-1$, 1). The time value vectors allow the model to take advantage of rule-based information.

\subsection{Combining Two Components}

We tried training the two components in a combined system, but found it slow to converge. In our experiments, we trained the pairwise model first, froze it, and then combined it with the GCL layer to train the GCL. This method also helps us observe whether the GCL component alone improves results, given the same input.

We tried combining the systems in two ways. One is to connect the output layer of the pretrained model to GCL, and the other is to slice the pre-trained model and connect its hidden layer to GCL. All the GCL layers are bi-directional, averaging forward and backward passes. By connecting the output layer, which has a softmax activation, we hand the final decisions made by the pairwise model to GCL. On the other hand, the hidden layer provides higher layers with cruder but richer information. We found that the latter performs better. It is also possible to train the two components together from scratch. In this case, the learning rate has to be set much lower to assure convergence, and the training requires more epochs.

\section{Experiments}

For all the experiments, hyperparameters including the number of epochs are tuned with the validation set only. Training data is segmented into chunks. Each chunk contains relation pairs in the narrative order. The size of chunks is randomly chosen from $[40,60,80,120,160]$ at the beginning of each epoch of training. The GCL maintains a memory for each chunk, and clears it at the end of a chunk. The idea here is to train the model on short paragraphs to avoid overfitting.

To introduce further randomness, the chunks are rotated for each epoch. For a specific training file, if chunk $i$ starts with pair $n_{i}$ in epoch 1 , in epoch 
2 , chunk $i$ will start with pair $n_{i}+$ chunksize +11 . 11 is a prime number we chose to assure each epoch observes different compositions of chunks. By doing the rotation, some pairs in the final chunk of epoch 1 will show up in the first chunk in epoch 2 as well. However, within each chunk, we do not randomize pairs, so narrative order is preserved at this level. We also do not shuffle the chunks, but only rotate them.

Evaluation on the test set uses only one chunk for each file (chunk size is the number of pairs). Each relation pair is only processed once, without "multiple rounds of reading". Thus, we essentially train the model to read shorter paragraphs (varied in length), but test it on long articles.

\subsection{Pairwise Model}

As described in Section 3.2, the pairwise classifier has the following input vectors: left and right shortest path branches, two flat context vectors, a pair type flag, and a time value vector. Word embeddings are initialized with glove.840B.300d word vectors ${ }^{2}$, and set to be trainable. The BiLSTM layers are followed by max-pooling. The two hidden layers have size 512 and 128, respectively. We train this model for 40 epochs, using the RMSProp optimizer (Tieleman and Hinton, 2012). The learning rate is scheduled as $l r=2 \times 10^{-3} \times 2^{-\frac{n}{5}}$, where $n$ is the number of epochs.

The middle block of Table 1 shows the performance of the pairwise model after applying double-checking. Since all pairs are flipped, double-checking combines results from $\left(e_{i}, e_{j}\right)$ and $\left(e_{j}, e_{i}\right)$, picking the label with the higher probability score, which typically boosts performance. The results without double-checking show similar trends.

\subsection{GCL model}

After training the pairwise model, we combine it with GCL. Unless otherwise indicated, the results reported in this section use the model configuration that connects the hidden layer (rather than the output layer) of the pairwise model with a bidirectional GCL layer. The bidirectional GCL is realized as the average of a forward GCL and a backward GCL, each producing a sequence. Then two more hidden layers are put on top of it, followed

\footnotetext{
${ }^{2}$ https://nlp.stanford.edu/projects/glove/

${ }^{3}$ This result does not include timex-timex pairs, which is $3 \%$ of total test instances.
}

\begin{tabular}{|l|c|c|}
\hline Model & Micro-F1 & Macro-F1 \\
\hline CAEVO (not NN model) & .507 & \\
CATENA (not NN model) & .511 & \\
Cheng et al. 2017 & $.520^{3}$ & \\
Meng et al. 2017 & & .519 \\
\hline pairwise & .535 & .528 \\
\hline Two more hidden layers & .539 & .532 \\
GCL w/ state-tracking controller & .545 & $\mathbf{. 5 3 8}$ \\
GCL w/ stateless controller & $\mathbf{. 5 4 6}$ & $\mathbf{. 5 3 8}$ \\
GCL w/ pre-trained output layer & .541 & .536 \\
\hline
\end{tabular}

Table 1: Results on the test set. The GCL models use the same hyperparameters, if possible. The two models on the top do not use neural networks. The results in the two lower blocks all use double-check. "Two more hidden layers" means adding two dense layers on top of the pre-trained model without using GCL. The last row corresponds to connecting the output layer of a pre-trained model to GCL layers with stateless controller.

by an output layer. All the layers in the pre-trained pairwise model are set to be untrainable. The two trainable hidden layers have sizes 512 and 128, respectively, with ReLU activtion and 0.3 dropout after each one. The GCLs have 128 memory slots. Learning rate is scheduled as $l r=2 \times 10^{-4} \times 2^{-\frac{n}{2}}$. In the experiments, we found the models converge quite fast with respect to the number of epochs. It is not surprising because the lower layers are already well trained, and frozen (no updating). After the 5th epoch, the training accuracy typically reaches 0.95 . We stop training after 10 epochs.

The bottom block of Table 1 presents the results, showing that all models from the present paper outperform existing models from the literature. One may argue the combined system adds more hidden layers over a pre-trained model, which contributes to the improvement in performance. We show a comparison to a baseline model which adds two dense layers on top of the pairwise model, without the GCL. The configuration of the two layers is the same as we used for the GCL models. The result shows that the performance is slightly higher than what we get from the pairwise model, but the difference is smaller than what we get from GCL models - suggesting that the performance improvement with GCL models is not just due to more parameters. We also tried adding an LSTM layer on top of the pre-trained model, and found the system cannot converge. It again confirms that GCL is more powerful than LSTM in handling irregular time series.

We found no difference in performance between the stateless controller and state-tracking controller. Connecting the output layer of the pre- 
trained model to GCL seems to generate weaker results than connecting the hidden layer, although it also outperforms the pairwise model, and all previous models in literature.

We performed significance testing to compare the pairwise model and the GCL-enabled model. A paired one-tailed t-test shows the results from the GCL model are significantly higher than results from pairwise model (p-value 0.0015). While significant, the improvement is relatively small, we believe due in part to the small size of Timebank-Dense dataset.

\subsection{Case Study}

To illustrate the difference in performance of the pairwise model and the GCL model, we created a sample paragraph in which long-distance dependencies and references to DCT are needed to resolve some of the temporal relations:

John met Mary in Massachusetts when they attended the same university. They are getting married in 2019 , 2 years after their graduation. But this year, they have relocated to New Hampshire.

We created the gold standard annotation for this text with 5 events, 2 timexes, and 24 TLINKs (see appendix $)^{4}$. We set the DCT to an arbitrary date "2018-04-01". There are no VAGUE or SIMULTANEOUS relations.

For this paragraph, the pairwise model yields an accuracy (i.e. micro-averaged F1) of 0.292, while the GCL-enabled model yields 0.417. Overall, the GCL-enabled model assigns 6 VAGUE labels while the pairwise assigns 11 . It reflects the fact that GCL tries to infer relations from otherwise vague evidence. For example, it is difficult to infer the relation between met and 2019 from the local context (without DCT, particularly), so the pairwise model labels it as VAGUE, while the GCL-enabled model correctly assigns BEFORE.

Recall that the GCL is placed on top of a pretrained pairwise model, so the mistakes made by the pairwise model propagate to GCL. For example, the pairwise model incorrectly classifies 2019 as BEFORE graduation - perhaps, due to a somewhat unusual syntax. But the GCL-enabled system assigns it a VAGUE label, probably as a way to compromise. In the TimeBank-Dense test data, VAGUE cases dominate, which may have made it more difficult for GCL to assign proper labels. In the future, we believe it may be better to omit

\footnotetext{
${ }^{4}$ Note that in TimeBank-Dense, no TLINKS are associated DURATION timexes, so 2 years is not annotated
}

writing (and reading) the VAGUE relations to/from GCL.

\subsection{Error Analysis}

Table 2 shows the overall performance for each relation using the GCL system with the stateless controller. Since we flip pairs and use doublechecking to pick one result for each pair, BEFORE/AFTER and IS_INCLUDED/INCLUDES are actually treated in the same way, respectively. Here we map the results to original pairs, in order to compare to other systems.

\begin{tabular}{|c|c|c|c|c|c|c|c|}
\hline & \multicolumn{6}{|c|}{ Predicted labels } & \multirow[b]{2}{*}{ Total } \\
\hline & SIMUL & BEF & AFT & IS_INCL & INCL & VAG & \\
\hline SIMUL & 10 & 0 & 9 & 2 & 1 & 17 & 39 \\
\hline BEF & 0 & 327 & 27 & 15 & 5 & 215 & 589 \\
\hline AFT & 1 & 26 & 208 & 4 & 5 & 184 & 428 \\
\hline IS_INCL & 1 & 27 & 3 & 59 & 2 & 67 & 159 \\
\hline INCL & 0 & 16 & 9 & 2 & 19 & 70 & 116 \\
\hline VAG & 1 & 171 & 87 & 28 & 17 & 596 & 900 \\
\hline
\end{tabular}

Table 2: Overall results per relation.

As the table shows, the VAGUE relation causes the most trouble. It is not only because VAGUE is the largest class, but also because it is often semantically ambiguous, so even human experts have low inter-annotator agreement. If we allow a relatively sparse labeling of data, and use other evaluation methods (e.g. question answering), the VAGUE class is not likely to have similar effects.

We also break down the results according to the types of pairs. Compared to other systems, our approach has a big advantage for event-event (EE) pairs, which is by far the most common (64\%) relation pairs for all data, and also requires more complex natural language understanding. Com-

\begin{tabular}{|l|ccc|l|}
\hline Systems & E-D & E-E & E-T & Overall \\
Frequency & $14 \%$ & $64 \%$ & $19 \%$ & $97 \%$ \\
\hline CAEVO & $\mathbf{. 5 5 3}$ & .494 & $\mathbf{. 4 9 4}$ & .502 \\
CATENA & .534 & .519 & .468 & .512 \\
Cheng et al. 2017 & .546 & .529 & .471 & .520 \\
\hline GCL & .489 & $\mathbf{. 5 7 0}$ & .487 & $\mathbf{. 5 4 2}$ \\
\hline
\end{tabular}

Table 3: Results on the E-D, E-D and E-T pairs. GCL stands for the GCL-enabled system with a stateless controller. Frequencies are percentages in the test set. T-T pairs are not shown here. CAEVO is from Chambers et al. (2014). CATENA is from Mirza and Tonelli (2016)

paired to CAEVO, our performance on event-DCT (E-D) and event-timex (E-T) pairs is not that great. CAEVO uses engineered features such as entity attributes, temporal signals, and semantic information from WordNet, which seems to work well in these two cases. We took a closer look at our E-D 
results, and found that the relatively low performance is mainly caused by misclassifying VAGUE as AFTER. As Table 4 shows, among the 72

\begin{tabular}{|l|cccccc|}
\hline & \multicolumn{7}{|c|}{ Predicted labels } \\
& SIMUL & BEF & AFT & IS_INCL & INCL & VAG \\
\hline SIMUL & 0 & 0 & 0 & 0 & 0 & 0 \\
BEF & 0 & 57 & 11 & 15 & 6 & 37 \\
AFT & 0 & 3 & 36 & 0 & 0 & 10 \\
IS_INCL & 0 & 11 & 1 & 31 & 1 & 12 \\
INCL & 0 & 0 & 2 & 1 & 3 & 2 \\
VAG & 0 & 4 & 20 & 9 & 14 & 25 \\
\hline
\end{tabular}

Table 4: Test results from event and document creation time (E-D) pairs. The rows are true labels and the columns are predicted labels.

VAGUE relations in E-D pairs, 20 are labeled AFTER by our system. In a news article, most events occur before the DCT i.e. the time when the article was written. If the temporal relation is vague, our system tends to guess that the event occurs after the DCT. It is interesting because AFTER only accounts for $16 \%$ of all E-D pairs in test data (and about the same in training data), behind BEFORE (41\%), VAGUE (21\%), and IS_INCLUDED (18\%). However, E-D is a relatively small category with only 311 instances in the test set, so it is difficult to draw any a substantive conclusion in this case.

Recall that our model has a uniform architecture for all input types and is trained on eventevent, event-timex and event-DCT pairs simultaneously. As a result, its performance is not optimal for some lower-frequency pair types. Tuning the model for each pair type separately, as well as resampling to deal with class imbalance would, perhaps, improve performance. However, the point of these experiments was not to get the largest improvement, but to show that the GCL mechanism can replace heuristic-based timegraph conflict resolution, improving the performance of an otherwise very similar model.

\section{Related Work}

While the GCL model is inspired by NTM, other NTM variants have also been proposed recently. Zhang et al. (2015) proposed structured memory architectures for NTMs, and argue they could alleviate overfitting and increase predictive accuracy. Graves et al. (2016) proposed a memory access mechanism on top of NTM, which they call Differentiable Neural Computer (DNC). DNC can store the transitions between memory locations it accesses, and thus can model some structured data.

Gülçehre et al. (2016) proposed a Dynamic Neural Turing Machine (D-NTM) model, which allows discrete access to memory. Gülçehre et al. (2017) further simplified the addressing algorithm, so a single trainable matrix is used to get locations for read and write. Both models separate the address section from the content section of memory, as do we. We came up with the idea independently, noting that the content-based addressing in the canonical NTM model is difficult to train. A crucial difference between GCL and these models is that they use input "content" to compute keys. In GCL, the addressing mechanism fully depends on the entity representations, which are provided by the context encoding layers and not computed by the GCL controller. Addressing then involves matching the input entities and the entities in memory.

Other than NTM-based approaches, there are models that use an attention mechanism over either input or external memory. For instance, the Pointer Networks (Vinyals et al., 2015) uses attention over input timesteps. However, it has no power to rewrite information for later use, since they have no "memory" except for the RNN states. The Dynamic Memory Networks (Kumar et al., 2016) has an "episodic memory" module which can be updated at each timestep. However, the memory there is a vector ("episode") without internal structure, and the attention mechanism works on inputs, just as in Pointer Networks. Our GCL model and other NTM-based models have a memory with multiple slots, and the addressing function (attention) dictates writing and reading to/from certain slots in the memory.

\section{Conclusion}

We have proposed the first context-aware neural model for temporal information extraction using an external memory to represent global context. Our model introduces a Global Context Layer which is able to save and retrieve processed temporal relations, and then use this global context to infer new relations from new input. The memory can be updated, allowing self-correction. Experimental results show that the proposed model beats previous results without resorting to ad-hoc resolution of timegraph conflicts in postprocessing.

\section{Acknowledgments}

This project is funded in part by an NSF CAREER award to Anna Rumshisky (IIS-1652742). 


\section{References}

Nathanael Chambers, Taylor Cassidy, Bill McDowell, and Steven Bethard. 2014. Dense event ordering with a multi-pass architecture. Transactions of the Association for Computational Linguistics, 2:273284.

Fei Cheng and Yusuke Miyao. 2017. Classifying temporal relations by bidirectional lstm over dependency paths. In $A C L$.

Jason Alan Fries. 2016. Brundlefly at semeval-2016 task 12: Recurrent neural networks vs. joint inference for clinical temporal information extraction. CoRR, abs/1606.01433.

Alex Graves, Greg Wayne, and Ivo Danihelka. 2014. Neural turing machines. CoRR, abs/1410.5401.

Alex Graves, Greg Wayne, Malcolm Reynolds, Tim Harley, Ivo Danihelka, Agnieszka GrabskaBarwinska, Sergio Gomez Colmenarejo, Edward Grefenstette, Tiago Ramalho, John Agapiou, Adrià Puigdomènech Badia, Karl Moritz Hermann, Yori Zwols, Georg Ostrovski, Adam Cain, Helen King, Christopher Summerfield, Phil Blunsom, Koray Kavukcuoglu, and Demis Hassabis. 2016. Hybrid computing using a neural network with dynamic external memory. Nature, 538(7626):471476.

Çaglar Gülçehre, Sarath Chandar, and Yoshua Bengio. 2017. Memory augmented neural networks with wormhole connections. CoRR, abs/1701.08718.

Çaglar Gülçehre, Sarath Chandar, Kyunghyun Cho, and Yoshua Bengio. 2016. Dynamic neural turing machine with soft and hard addressing schemes. CoRR, abs/1607.00036.

Sepp Hochreiter and Jürgen Schmidhuber. 1997. Long short-term memory. Neural Computation, 9(8):1735-1780.

Ankit Kumar, Ozan Irsoy, Peter Ondruska, Mohit Iyyer, James Bradbury, Ishaan Gulrajani, Victor Zhong, Romain Paulus, and Richard Socher. 2016. Ask me anything: Dynamic memory networks for natural language processing. In Proceedings of The 33rd International Conference on Machine Learning, volume 48 of Proceedings of Machine Learning Research, pages 1378-1387, New York, New York, USA. PMLR.

Chen Lin, Timothy A. Miller, Dmitriy Dligach, Steven Bethard, and Guergana Savova. 2017. Representations of time expressions for temporal relation extraction with convolutional neural networks. In BioNLP 2017, Vancouver, Canada, August 4, 2017, pages 322-327.

Xiao Ling and Daniel S. Weld. 2010. Temporal information extraction. In Proceedings of the TwentyFourth AAAI Conference on Artificial Intelligence, AAAI 2010, Atlanta, Georgia, USA, July 11-15, 2010.
Inderjeet Mani, Ben Wellner, Marc Verhagen, and James Pustejovsky. 2007. Three approaches to learning tlinks in timeml. Technical Report CS-07268, Computer Science Department.

Yuanliang Meng, Anna Rumshisky, and Alexey Romanov. 2017. Temporal information extraction for question answering using syntactic dependencies in an 1stm-based architecture. In Proc. of the conference on empirical methods in natural language processing (EMNLP).

P Mirza and S Tonelli. 2016. Catena: Causal and temporal relation extraction from natural language texts. In The 26th International Conference on Computational Linguistics, pages 64-75. Association for Computational Linguistics.

Paramita Mirza and Anne-Lyse Minard. 2015. Hlt-fbk: a complete temporal processing system for qa tempeval. In Proc. of the 9th International Workshop on Semantic Evaluation (SemEval 2015), pages 801805. Association for Computational Linguistics.

Weiyi Sun. 2014. Time Well Tell: Temporal Reasoning in Clinical Narratives. PhD dissertation. Department of Informatics, University at Albany, SUNY.

Weiyi Sun, Anna Rumshisky, and Ozlem Uzuner. 2013. Evaluating temporal relations in clinical text: 2012 $\mathrm{i} 2 \mathrm{~b} 2$ challenge. Journal of the American Medical Informatics Association, 20(5):806-813.

T Tieleman and G Hinton. 2012. Lecture 6.5-rmsprop: Divide the gradient by a running average of its recent magnitude. COURSERA: Neural networks for machine learning, 4(2):26-31.

Julien Tourille, Olivier Ferret, Aurelie Neveol, and Xavier Tannier. 2017. Neural architecture for temporal relation extraction: A bi-lstm approach for detecting narrative containers. In Proceedings of the 55th Annual Meeting of the Association for Computational Linguistics (Volume 2: Short Papers), pages 224-230, Vancouver, Canada. Association for Computational Linguistics.

Oriol Vinyals, Meire Fortunato, and Navdeep Jaitly. 2015. Pointer networks. In C. Cortes, N. D. Lawrence, D. D. Lee, M. Sugiyama, and R. Garnett, editors, Advances in Neural Information Processing Systems 28, pages 2692-2700. Curran Associates, Inc.

Katsumasa Yoshikawa, Sebastian Riedel, Masayuki Asahara, and Yuji Matsumoto. 2009. Jointly identifying temporal relations with markov logic. In Proceedings of the Joint Conference of the 47th Annual Meeting of the ACL and the 4th International Joint Conference on Natural Language Processing of the AFNLP: Volume 1 - Volume 1, ACL '09, pages 405413, Stroudsburg, PA, USA. Association for Computational Linguistics.

Wei Zhang, Yang Yu, and Bowen Zhou. 2015. Structured memory for neural turing machines. CoRR, abs/1510.03931. 